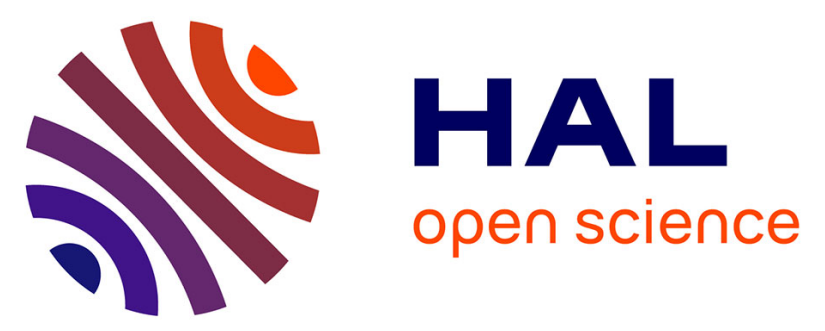

\title{
Electrostatic Embedding To Model the Impact of Environment on Photophysical Properties of Molecular Crystals: A Self-Consistent Charge Adjustment Procedure
}

Liam Wilbraham, Carlo Adamo, Fredericfredefrederic Labat, Ilaria Ciofini

\section{To cite this version:}

Liam Wilbraham, Carlo Adamo, Fredericfredefrederic Labat, Ilaria Ciofini. Electrostatic Embedding To Model the Impact of Environment on Photophysical Properties of Molecular Crystals: A SelfConsistent Charge Adjustment Procedure. Journal of Chemical Theory and Computation, 2016, 12, pp.3316 - 3324. 10.1021/acs.jctc.6b00263 . hal-01534248

\section{HAL Id: hal-01534248 \\ https: / hal-enscp.archives-ouvertes.fr/hal-01534248}

Submitted on 13 Jun 2017

HAL is a multi-disciplinary open access archive for the deposit and dissemination of scientific research documents, whether they are published or not. The documents may come from teaching and research institutions in France or abroad, or from public or private research centers.
L'archive ouverte pluridisciplinaire HAL, est destinée au dépôt et à la diffusion de documents scientifiques de niveau recherche, publiés ou non, émanant des établissements d'enseignement et de recherche français ou étrangers, des laboratoires publics ou privés. 


\title{
Electrostatic Embedding To Model the Impact of Environment on Photophysical Properties of Molecular Crystals: A Self-Consistent Charge Adjustment Procedure
}

\author{
Liam Wilbraham, ${ }^{\dagger}$ Carlo Adamo, ${ }^{\dagger, \dagger}$ Frédéric Labat, ${ }^{*}{ }^{\dagger}$ and Ilaria Ciofini ${ }^{*}, \dagger$ \\ ${ }^{\dagger}$ PSL Research University, Institut de Recherche de Chimie Paris IRCP, CNRS-Chimie ParisTech, 11 rue Pierre et Marie Curie, \\ F-75005 Paris, France \\ ${ }^{\ddagger}$ Institut Universitaire de France, 103 Boulevard Saint Michel, F-75005 Paris, France
}

\author{
Supporting Information
}

ABSTRACT: A case study of 1,8-dihydroxy-2-napthaldehyde (DHNA) - exhibiting an excited-state intramolecular double proton transfer resulting in photophysical properties sensitive to the surrounding environment-has been used to assess the performance of electrostatic embedding approaches designed to accurately recover the effects of a bulk crystalline environment on calculated photophysical properties. The first approach, based on time-dependent density functional theory (TD-DFT) applied in a $\mathrm{QM} / \mathrm{QM}^{\prime}$ scheme, makes use of a

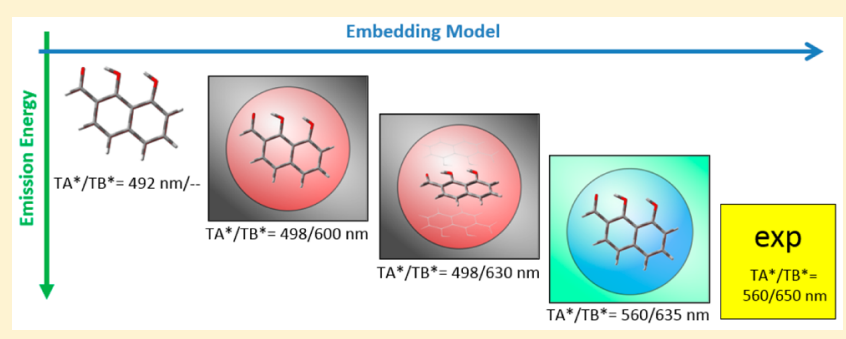
background point charge distribution which can accurately reproduce the exact ground-state Ewald potential of the bulk crystal. The second approach seeks to "optimize" these charges in a self-consistent manner in order to reproduce the electrostatic field produced by the environment at the excited state. Using these two approaches, both absorption and emission properties of molecular crystals, such as the position and the relative shift in the emission bands in the solid state with respect to solution, can be accurately reproduced. More generally, the results obtained show how these computationally affordable approaches can be used to predict the excited-state behavior of molecules in condensed phases, thus allowing their employment to predict or design new molecular materials with enhanced photophysical properties.

\section{INTRODUCTION}

It is well-known that photophysical properties of a given molecular system can be strongly influenced, and are often predicated, by interactions with the surrounding environment, whether it be surrounding solvent or indeed other surrounding molecules within a molecular crystal. $^{1-5}$ For this reason, considerable resources have been dedicated to the study of environmental influence in order to gain insight into, and control over, different excited-state processes, which can give rise to potentially useful photophysical traits for application in optoelectronic devices. $^{6-9}$

From both an experimental and theoretical perspective, methods for studying photophysical properties in solvents are very well developed, ${ }^{10-12}$ such that it is becoming possible to gain control over and tune excited-state processes in solution. Excited-state processes are more difficult to characterize experimentally in the solid state and reports of interesting photophysical behavior in periodic systems, which can often be small and subtle, are far more rare, when compared with solution.

From a theoretical point of view, methods for studying photophysical properties in crystals are far less developed, although some strategies already exist both using Gaussian-type orbitals (GTOs) ${ }^{13-15}$ or projector augmented wave (PAW) as a basis. $^{16,17}$
Nevertheless, the study of photophysical properties of molecular crystals is, in current practice, exclusively limited to procedures based on the "extraction" of clusters from an optimized periodic structure, which can then be treated at different quantum levels of theory (with an associated spectrum of computational cost). These approaches often rely on multilayer methods with a central molecule of interest embedded by its surrounding neighbors treated either at quantum level $\left(\mathrm{QM} / \mathrm{QM}^{\prime}\right),{ }^{6,18}$ or classically (QM/MM). ${ }^{19}$

While these methods are often successful, they ignore the potential effects that the periodic nature of the crystal might have on its photophysical properties. For this reason, we will attempt to incorporate these effects by embedding these aforementioned clusters in an array of point charges, which generate the exact electrostatic field of the infinite crystal. The idea of incorporating such a field is, in itself, nothing new; ${ }^{20-24}$ however, to the best of our knowledge, it has not yet been applied for the study of excited-state properties of molecular crystals. Here, we propose the method developed by Derenzo and co-workers, ${ }^{25}$ initially intended for studying localized structural defects in ionic crystals, to study excited-state processes in these periodic systems.

Received: March 14, 2016

Published: May 27, 2016 
We aim to use a case study of a crystalline system exhibiting a double proton transfer reaction yielding dual emission behavior to show that (i) we can use an algorithm to inexpensively and easily incorporate these interactions into any crystalline model with 3D periodicity for the study of its photophysical properties and (ii) their inclusion leads to a better reproduction of the environmental effects that are imposed by the crystalline environment and therefore better insight into how these might affect relevant photophysical processes. At this point, we would like to stress the difference between this approach and more "conventional" approaches to charge embedding. While more traditional approaches indeed seek to incorporate effects of the surrounding crystalline medium with often large arrays of point charges fixed at predefined values obtained from cluster calculations, these do not reproduce the exact coulomb potential of a crystalline system. This is due to the charge array inevitably being truncated-regardless of its size-and the concomitant loss of the effect of infinite periodicity on the electrostatic potential. In contrast, this work seeks to use a fitting process that imposes the exact coulomb potential of the infinite crystal within a predefined area of interest.

The choice of our test case was motivated by the fact that, in recent years, solid-state emitters based on the excited-state intramolecular proton transfer (ESIPT) reaction have attracted intense interest in the field of optoelectronic devices, as a result of their sought-after and unique photophysical properties. ${ }^{2}$ However, solid-state devices that take advantage of the ESIPT mechanism suffer notable shortcomings, including low quantum yield and short fluorescence lifetimes. In order to aid their development, a better understanding of the mechanisms at play at the excited state is crucial. Moreover, these mechanisms are challenging to analyze from an experimental point of view, highlighting the importance of theoretical approaches in their study. From previous theoretical studies on model ESIPT compounds, the proton transfer mechanism results from an excitation with limited chargetransfer character, resulting in increased acidity (basicity) of the proton donor (acceptor) site, and the subsequent transfer of the proton as the molecule is allowed to relax on the excitedstate potential energy surface. ${ }^{26,27}$

With this in mind, we have chosen, as an initial case study, the recently synthesized molecular crystal comprised of 1,8dihydroxy-2-napthaldehyde (DHNA), ${ }^{28}$ which is schematically depicted in Figure 1. This molecule was strategically designed

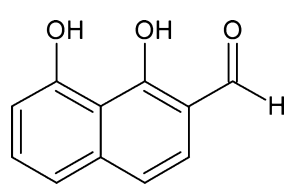

Figure 1. Structure of the 1,8-dihydroxy-2-napthaldehyde (DHNA) molecule ${ }^{28}$ considered in this work.

to directly probe the excited-state intramolecular double proton transfer (ESIDPT) mechanism, which arises from two distinct intramolecular hydrogen bonds and results in clear dual emission. Peng and co-workers ${ }^{28}$ conducted a comprehensive experimental investigation of the photophysical properties of DHNA in cyclohexane solution and in single crystalline form with an accompanying theoretical analysis, in the case of the solution, performed using density functional theory (DFT) and time-dependent density functional theory (TD-DFT). In the case of DHNA in cyclohexane solution, using ultraviolet- visible light (UV-vis) spectroscopy and time-resolved femtosecond fluorescence upconversion techniques, they were able to attribute the observed dual emission to radiation from species that arose after the first $\left(\mathrm{TA}^{*}\right)$ and second $\left(\mathrm{TB}^{*}\right)$ proton transfer reaction. A general outline of the proposed mechanism is thus depicted in Figure 2. Interestingly, going from solution to the solid state, the $\mathrm{TA}^{*}$ emission band undergoes a significant red shift-from $520 \mathrm{~nm}$ to $560 \mathrm{~nm}$ - while the position of the $\mathrm{TB}^{*}$ emission band remains unchanged.

Furthermore, insight into the thermodynamics of the ESIDPT processes in both the solution and the single crystal was gleaned from the study of variable-temperature emission spectra, with interesting results. In the case of the solution, the $\mathrm{TB}^{*}$ state is thermodynamically favored, whereas, in the solid state, the TA* state is prevalent. This stark difference between the thermodynamics of the ESIDPT reaction in the solid state and in solution highlights the sensitivity of this reaction to environmental effects. These differences, associated with an environmental change from solution to solid state, make this system an ideal case study for this method.

The paper is structured as follows: following an outline of the computational details, a comparison of the different models used in this work is given. Next, the reproduction of the changes in the dual emission phenomenon between solvated and solid-state DHNA is discussed, first for the simple charge embedding applied in a $\mathrm{QM} / \mathrm{QM}^{\prime}$ scheme and then for its selfconsistent variant to simulate its emissive behavior. Finally, some concluding remarks are given.

\section{COMPUTATIONAL DETAILS}

The computational procedure involves calculations that make use of single molecules and molecular clusters, which are predicated by calculations using periodic boundary conditions. In this work, we employ only density functional theory (DFT) for ground-state calculations and time-dependent density functional theory (TD-DFT) for those at the excited state.

All calculations using periodic boundary conditions were performed with the Crystal code, ${ }^{29}$ which makes use of allelectron, atom-centered Gaussian basis sets. Energy convergence, with respect to the number of $k$-points in the Monkhorst-Pack grid, was confirmed with the number of $k$ points in the irreducible Brillouin zone set to 30 . The default settings for integral tolerances and convergence criteria were used. A full geometry optimization was conducted at the B3LYP-D2 level of theory, ${ }^{30-32}$ which introduces empirical corrections to account for dispersion interactions, ${ }^{33}$ with a double- $\zeta$ plus polarization plus diffuse basis set (i.e., the 6$31+G(d, p)$ Pople basis). For solid-state calculations, diffuse functions were omitted from the basis set due to problems related to basis set linear dependence. As well as the geometry optimization, a population analysis was performed in order to determine the Mulliken charges for each atom in the unit cell.

Using this optimized geometry and the associated Mulliken charges, the Ewald code ${ }^{34}$ was used to construct and compute an array of background charges. We will give a brief summary of how the algorithm works, but we direct the reader to the original paper for full details of the method. This algorithm seeks to reproduce the Ewald potential felt by a predefined cluster within a $3 \mathrm{D}$ periodic lattice using only the knowledge of the positions and partial charges of the atoms within the crystallographic unit cell. In order to accomplish this accurately, an $N_{x} \times N_{y} \times N_{z}$ supercell is formed, which is then split into three zones: (1) the cluster of interest, which will be given 


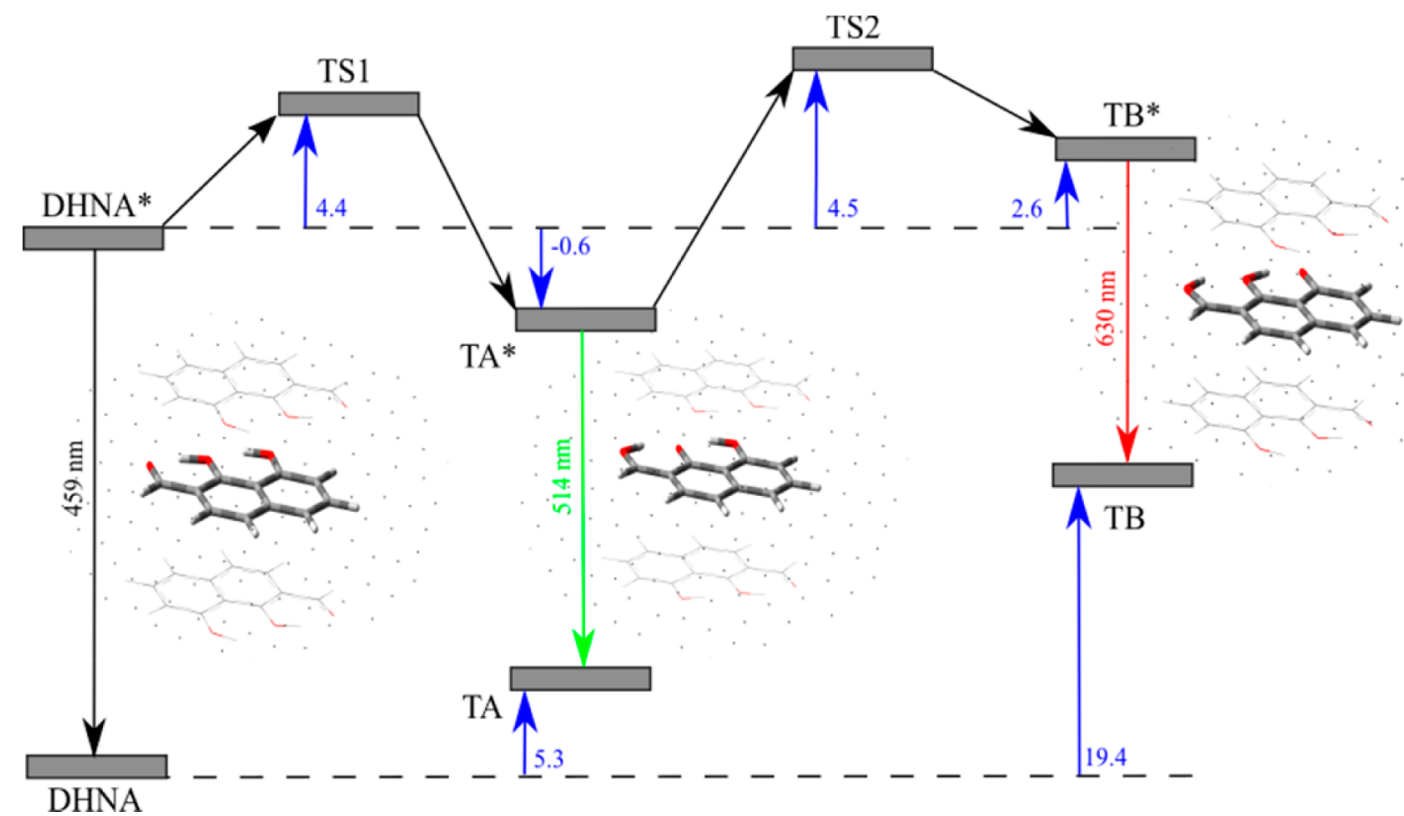

Figure 2. General reaction scheme for the excited-state intramolecular double proton transfer (ESIDPT) reaction taking place in crystalline DHNA. The values here are those computed using model $T_{\mathrm{EMB}}$ (see Figure 5, presented later in this work) with electronic embedding. Emission wavelengths (in $\mathrm{nm}$ ) and relative energies with respect to DHNA (in $\mathrm{kcal} / \mathrm{mol}$ ) are given. Those shown for the ground-state species correspond to points calculated on the ground-state potential energy surface at the corresponding optimized excited-state geometry.

quantum mechanical treatment; (2) a spherical zone of point charges encasing the first zone, with its partial charge values being held constant; and (3) all other point charges, which are allowed to vary (Figure 3 ). In the charge-fitting process, the

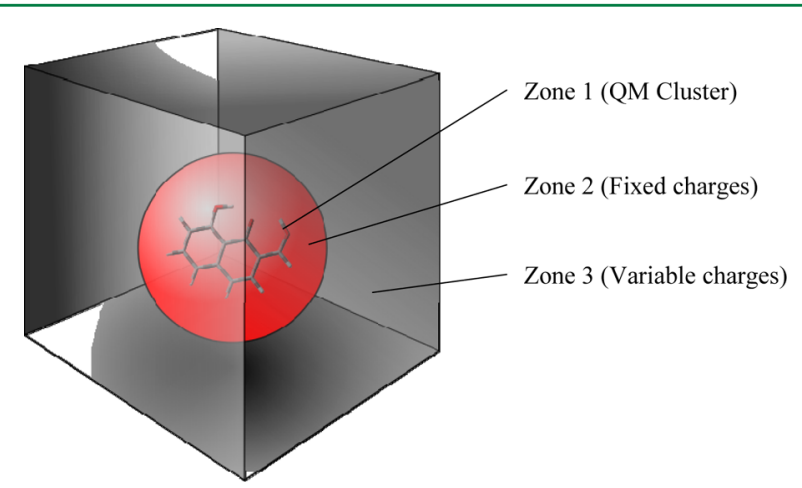

Figure 3. Schematic diagram of the simplest model generated by employing the Ewald code. The quantum cluster (zone 1, molecule (here, the optimized $\mathrm{TA}^{*}$ form is shown)), point charges with fixed values (zone 2 , red sphere) and variable point charges (zone three, gray cube) are shown. Note this diagram gives no indication of the relative size of each zone and is not to scale. In reality, the ratio of the number of atoms in zones 2 and 3 is $\sim 1: 8$.

Ewald formula is used to compute the site potentials at all sites within zones 1 and 2 and, subsequently, a system of linear equations is solved to find charge values for zone 3 that allow the potential of all sites in zones 1 and 2 to be exactly equal to their Ewald values (and drives the overall dipole moment of the charge array to zero). This strategy is applied in both static and self-consistent schemes (see below).

Some minor modifications were also made to the Ewald code in the same fashion as ref 22. Specifically, the neglecting of point charges with values $<0.01 \mathrm{le}^{-} \mid$from the output files was remedied by reducing this cutoff to $10^{-5} \mid \mathrm{e}^{-} \mathrm{I}$ and the program was also allowed to work with partial rather than formal integervalue charges.

In ref 25 , general guidelines are given regarding the strategy for choosing the size of each zone for computing ground-state potentials. Although their relative and absolute size appear to be system-dependent, one essential constraint is that the rootmean-square (rms) error of the discrepancy between the Ewald potential and the computed potential (after charge fitting) in zones 1 and 2 should be sufficiently small. In the same study, it was also found that Ewald potentials in zone 1 were improved both by increasing the number of atoms in zone 2 and the overall size of the charge array (comprising zones 1, 2, and 3). Keeping these general considerations in mind, in this work, a 6 $\times 6 \times 4$ supercell was used in order to obtain a cube-like distribution of point charges resulting in a total of $\sim 12000$ point charges, which is close to the number suggested in ref 25 . For the quantum cluster (zone 1), both a DHNA monomer and a trimer were used, with the number of atoms in zone 2 set to 1500 in order to ensure that the central molecule(s) are not close to the outside "wall" of the sphere comprising zone 2 . Indeed, this gave an adequate rms error on the Ewald potential within zone 1 of $0.17 \mu \mathrm{V}$ for the monomer and $6.5 \mu \mathrm{V}$ for the trimer-deemed within the desired accuracy required for the calculation of absorption and emission spectra. Furthermore, increasing the size of zone 2, with the total number of charges in the array fixed, lead to no appreciable variation.

All molecular calculations were performed using the Gaussian program ${ }^{35}$ and the same $(6-31+G(d, p))$ basis set. In the first approach, each of the species in the ESIDPT reaction were studied with four different models, specifically using: (i) a single molecule in vacuum $\left(\mathrm{M}_{\mathrm{VAC}}\right)$, (ii) a single molecule embedded in the charge distribution generated by Ewald $\left(\mathrm{M}_{\mathrm{EMB}}\right)$, (iii) a trimer under vacuum $\left(\mathrm{T}_{\mathrm{VAC}}\right)$, and (iv) a trimer embedded in the charge distribution generated by Ewald $\left(\mathrm{T}_{\mathrm{EMB}}\right)$ (see Figure 5, presented later in this work). For calculations involving a single molecule, the B3LYP exchange- 


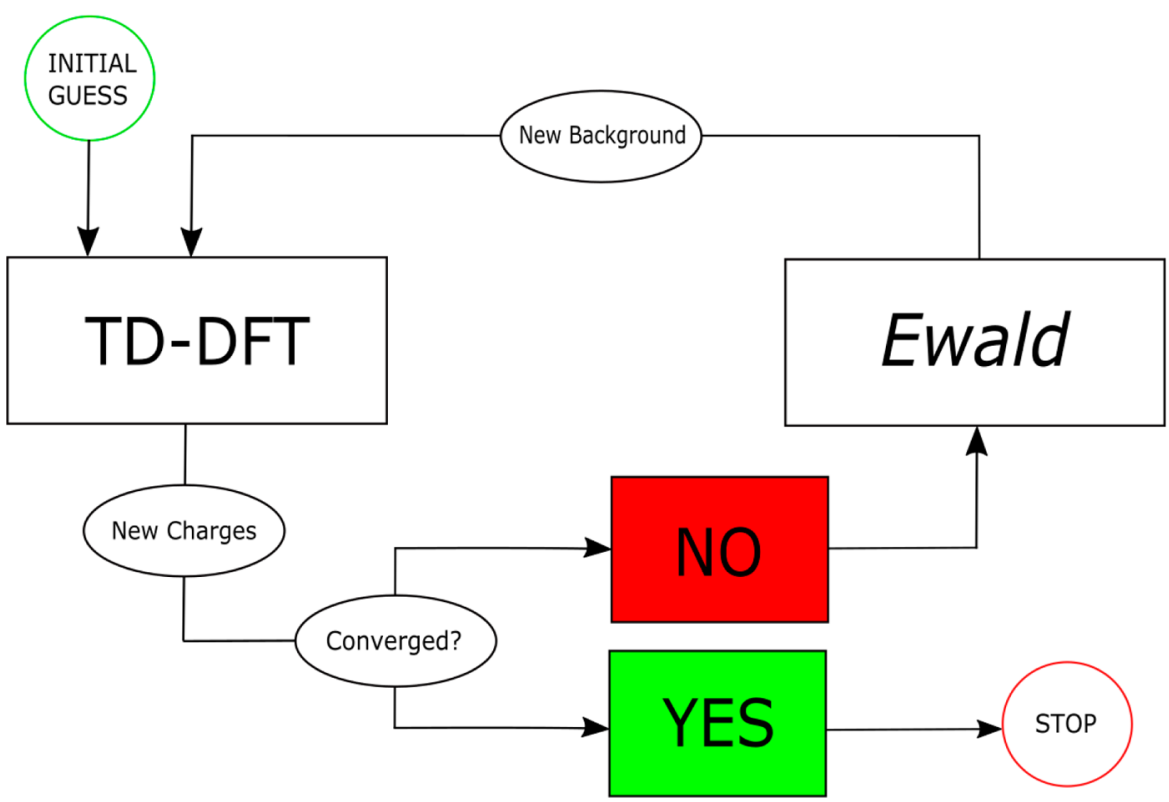

Figure 4. Schematic of the algorithm used to compute the charge background, which is self-consistent with the electron density of a given excited state of interest. The convergence criteria is the mean average deviation in calculated Mulliken charges of $<0.001 \mathrm{e}$, which was found to produce converged emission energies. The initial guess was that of the Mulliken charges determined using periodic boundary conditions.

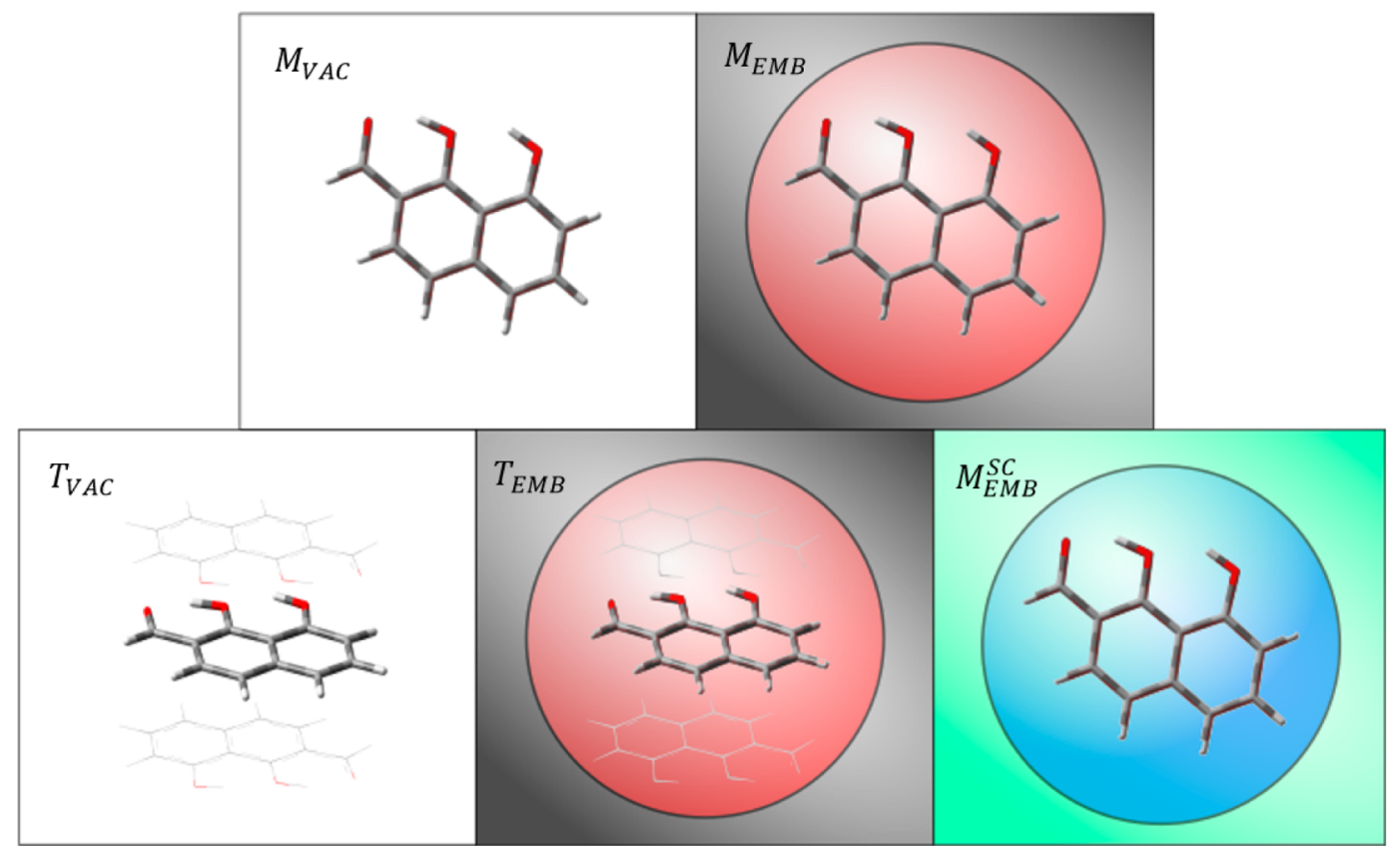

Figure 5. Schematic of models used in this work. The colored background signifies the presence of a background charge distribution generated by Ewald. Models include a single molecule in vacuum $\left(\mathrm{M}_{\mathrm{VAC}}\right)$, a single molecule embedded in the charge distribution generated by Ewald $\left(\mathrm{M}_{\mathrm{EMB}}\right)$, a trimer under vacuum $\left(\mathrm{T}_{\mathrm{VAC}}\right)$, and a trimer embedded in the charge distribution generated by Ewald $\left(\mathrm{T}_{\mathrm{EMB}}\right)$. The final model $\left(\mathrm{M}_{\mathrm{EMB}}^{\mathrm{SC}}\right)$ involves the same initial setup as model $\mathrm{M}_{\mathrm{EMB}}$, although the charge background is determined self-consistently with the excited state in question (indicated by the different colors of zones 2 and 3 ).

correlation functional was used to optimize the structure at the ground state for each of the species in the ESIDPT reaction. Subsequently, TD-DFT was used to optimize their structures at the excited state and calculate their respective emission energies. For calculations involving a trimer, the $\mathrm{ONIOM}^{36}$ $\mathrm{QM} / \mathrm{QM}^{\prime}$ method was employed using the B3LYP/6-31+G$(d, p)$ level of theory for the high layer and HF/STO-3G for the low layer, with the atoms in the low layer held fixed during the geometry optimization procedure. Each ONIOM calculation was performed with electronic embedding, in which the partial charges of the low level layer (and the Ewald charges, where appropriate) are incorporated into the quantum mechanical electronic Hamiltonian. As, in all cases, the point charge positions and low level ONIOM atoms are at the optimized ground-state geometry provided by the periodic calculations, it is assumed that there is no cooperativity between molecules during the ESIDPT reaction. 
Table 1. Computed Emission Wavelengths from the Lowest Lying Excited State of Each of the Species Involved in the ESIDPT Reaction $^{a}$

\begin{tabular}{|c|c|c|c|c|c|c|c|c|}
\hline \multirow[b]{2}{*}{ species } & \multicolumn{8}{|c|}{ Computed Emission Wavelengths for Each of the Species Involved in the ESIDPT Reaction (nm) } \\
\hline & $\mathrm{M}_{\mathrm{VAC}}$ & $\mathrm{M}_{\mathrm{SOL}}$ & $\mathrm{M}_{\mathrm{EMB}}$ & $\mathrm{T}_{\mathrm{VAC}}$ & $\mathrm{T}_{\mathrm{EMB}}$ & $\mathrm{M}_{\mathrm{EMBSC}}$ & solid state exp. & cyclohexane exp. \\
\hline DHNA* & & $\begin{array}{l}459 \\
(0.162)\end{array}$ & $\begin{array}{l}457 \\
(0.093)\end{array}$ & $\begin{array}{l}461 \\
(0.098)\end{array}$ & $\begin{array}{l}459 \\
(0.089)\end{array}$ & & & \\
\hline $\mathrm{TA}^{*}$ & $\begin{array}{l}492 \\
(0.127)\end{array}$ & $\begin{array}{l}498 \\
(0.174)\end{array}$ & $\begin{array}{l}498 \\
(0.116)\end{array}$ & $\begin{array}{l}505 \\
(0.122)\end{array}$ & $\begin{array}{l}514 \\
(0.115)\end{array}$ & $\begin{array}{l}560 \\
(0.085)\end{array}$ & 560 & 520 \\
\hline TB* & & 599 & 600 & 610 & 630 & 635 & 650 & 650 \\
\hline
\end{tabular}

${ }^{a}$ The corresponding calculated oscillator strengths, in arbitrary units (given in brackets). Abbreviations signify the type of model used, according to Figure 5 .

In the second approach, charges were recomputed selfconsistently with respect to the electron density for a given excited state, according to Figure 4. This was done in order to attempt to recover the mutual polarization effect between the molecule of interest and the surrounding crystal due to the electronic rearrangement that occurs upon excitation. Indeed this self-consistent procedure is expected to be necessary only when the environment has sufficient time to equilibrate, as in the case of emission.

This was done using a convergence criterion of $0.001 \mathrm{le}^{-} \mid$ with respect to the mean average deviation of the Mulliken charges of the central molecule, with tighter convergence criteria found to have a negligible effect on the computed emission energies. Note that, to avoid instabilities similar to those described previously, ${ }^{22}$ the background charges were computed self-consistently using a smaller basis $(6-31 G(d, p))$ and then the emission energies and new geometries calculated both using this basis set and by reintroducing the diffuse functions. A full set of results for the reduced basis set is given in the Supporting Information (Table S3), although we shall only discuss the results obtained using the full basis set in the main text. Also it is to be noted that, in this case, the asymmetric unit of the crystal structure is equal to that of a single molecule of DHNA. Employing the Ewald code in a selfconsistent manner, with respect to the charges computed quantum mechanically, was done previously in the determination of ground-state properties, such as NMR chemical shifts $^{22,23}$ and indeed, other embedding programs capable of accounting for local defects in crystalline environments in a selfconsistent manner exist. ${ }^{37}$

All minima were verified by computing the harmonic frequencies either analytically (ground state) or seminumerically (excited state) and checking that all frequencies were real. For transition states, one imaginary frequency, corresponding to the proton transfer reaction coordinate, was found.

\section{RESULTS AND DISCUSSION}

With the focus of this work being the prediction of excited-state properties in the solid state, we shall begin by discussing the performance of each model, (Figure 5) when attempting to reproduce the emission energy of each species observed via experiment. Table 1 shows all of the calculated emission wavelengths, given in nanometers, for each model studied in this work alongside the experimental data from both a single crystal and cyclohexane solution.

Generally, it can be seen that, at least at this level of theory, all models overestimate the emission energy of both the $\mathrm{TA}^{*}$ and $\mathrm{TB}^{*}$ species, with varying degrees of severity.
Properties of molecular crystals are often approximated via the study of an isolated molecule in the gas phase, assuming that all intermolecular forces are negligible. For this reason, we shall initially discuss the performance of each model, with respect to this approximation (designated here as $\mathrm{M}_{\mathrm{VAC}}$ ). In the case of $\mathrm{M}_{\mathrm{EMB}}$, the emission energy from $\mathrm{TA}^{*}$ is overestimated by a significant $0.31 \mathrm{eV}$. Furthermore, we were unable to achieve an excited-state minimum for the $\mathrm{TB}^{*}$ species, using only an isolated molecule, and so we were unable to calculate a meaningful emission energy. In turn, the absence of an excitedstate energy minimum for $\mathrm{TB}^{*}$ shows that this model fails to predict the inherent dual emission property of the DHNA molecular crystal observed experimentally. The rather large overestimation of the emission energy from $\mathrm{TA}^{*}$, together with the lack of a stable energy minimum for $\mathrm{TB}^{*}$, is the first indication of the importance of intermolecular effects in the DHNA molecular crystal and that their inclusion in any model is crucial to the prediction of the photophysical properties.

For model $\mathrm{M}_{\mathrm{EMB}}$, it is clear that the inclusion of the groundstate charge background offers a significant improvement. In the case of $\mathrm{TA}^{*}$ emission, the overestimation is reduced to 0.28 $\mathrm{eV}$, which, in any case, still represents a significant error. A more stark improvement can be found when analyzing the TB* emission. Not only was it possible to locate an energetic minimum on the excited state potential energy surface, the emission wavelength was predicted to be $600 \mathrm{~nm}$, in fairly good agreement with experiment, with an acceptable overestimation of $0.16 \mathrm{eV}$. In model $\mathrm{M}_{\mathrm{EMB}}$, we have attempted to accurately capture long-range electrostatic effects while ignoring other, likely influential, intermolecular forces. While the good agreement with the experimental $\mathrm{TB}^{*}$ emission is encouraging, the still rather poor agreement in the case of $\mathrm{TA}^{*}$ prompted us to further modify and refine the model in an attempt to capture other potentially important intermolecular effects.

This led to the introduction of models $T_{\mathrm{VAC}}$ and $\mathrm{T}_{\mathrm{EMB}}$ (Figure 5), in which we have designated a trimer of DHNA molecules to be treated at the quantum level. This was done in order to more accurately capture closer-range electrostatic effects each of the species in the ESDIPT reaction and lead to lower, and so more accurate, excitation energies.

In the case of model $\mathrm{T}_{\mathrm{EMB}}$, the inclusion of both the trimer and the charge background improves the prediction of the TA* emission wavelength to $514 \mathrm{~nm}$ (the best overall result, corresponding to an acceptable overestimation of $0.20 \mathrm{eV}$, with respect to experiment) while it has no effect on the calculated $\mathrm{TB}^{*}$ emission energy. Since this is the best performing model of those that we have proposed, with respect to experimental data, it highlights the effectiveness of both capturing the closer range electrostatic interactions while maintaining the longer- 
range electrostatic influence of the inherent periodicity of the crystal (described here in terms of the ground-state Ewald background charge distribution). The remaining error observed, with respect to the experimental data, other than inherent error in the exchange-correlation functional, could be ascribed to the nonpolarizable nature of the charge distribution. In fact, if the surrounding charge field were somehow allowed to equilibrate with the electronic density of the central molecule upon excitation, it could be reasonable to assume that this would further enhance the quality of the calculated emission energy of each species, which is a point that we shall revisit later.

Experimentally, the behavior of the ESIDPT reaction was observed to be rather different, depending on whether it takes place in cyclohexane solution or in a crystalline medium. Two major effects were observed: the apparent red shift of the TA* emission peak by $0.17 \mathrm{eV}(520-560 \mathrm{~nm})$ when going from the solution to the crystal and the inversion of relative emission band intensities of each species. Specifically, the emission band ascribed to the $\mathrm{TB}^{*}$ species is far more intense than that of the $\mathrm{TA}^{*}$ band in cyclohexane solution, with the inverse being true in the case of the single crystal.

Regarding the first effect, Peng and co-workers ${ }^{28}$ already conducted DFT and TD-DFT studies of the ESIDPT reaction using a single DHNA molecule in cyclohexane solution (designated $\mathrm{M}_{\mathrm{SOL}}$ ), taking advantage of a polarizable continuum model (PCM) to account for solvent effects. Therefore, we will use their results, in comparison with our calculated values of TA* emission, to assess each model's ability to reproduce the red shift (crystal shift) observed going from cyclohexane solution to the solid state.

It can be seen from Table 1 that model $\mathrm{M}_{\mathrm{EMB}}$ predicts no shift, with respect to the calculated value in cyclohexane solution, showing that the charge distribution alone is insufficient to model this effect. Moving to the trimer $\left(\mathrm{T}_{\mathrm{VAC}}\right)$, we make a modest improvement, the crystal shift at least traveling in the correct direction, with a calculated red shift of $0.03 \mathrm{eV}$. Next, considering model $\mathrm{T}_{\mathrm{EMB}}$, we see an improvement with respect to $\mathrm{T}_{\mathrm{VAC}}$ (crystal shift of $0.08 \mathrm{eV}$ ), bringing the calculated crystal shift into good agreement (within $0.1 \mathrm{eV}$ ) with the experimental value. However, in the same manner, the $\mathrm{TB}^{*}$ emission was determined to be $0.1 \mathrm{eV}$ lower in energy, with respect to the solution-even greater than that of $\mathrm{TA}^{*}-$ while no shift was observed experimentally for $\mathrm{TB}^{*}$. This shows that, at least for these models, the shift of the $\mathrm{TA}^{*}$ and $\mathrm{TB}^{*}$ emission in the solid state, with respect to solution, is not wellreproduced. This point will be reinvestigated within the context of the self-consistent charge scheme computed at the excited state.

With respect to the second effect-namely, the inversion of the relative intensities of the emission bands ascribed to TA* and $\mathrm{TB}^{*}$ going from cyclohexane solution to the crystal-we will first discuss the calculated oscillator strengths determined alongside each emission wavelength for the first four models shown in Figure 5, given in brackets in Table 1. We can see that, whether in solution or crystalline form and regardless of the model used to describe the crystalline environment, the calculated oscillator strength is slightly greater for $\mathrm{TB}^{*}$ than for TA*. This means that this information alone is insufficient to describe the intensity inversion phenomenon and points toward reaction thermodynamics as the root cause-as previously suggested by Peng and co-workers. ${ }^{28}$
To gain more insight, the excited-state potential energy surface was mapped out along the ESIDPT reaction coordinate via interpolation between minimal energy structures at the excited state for models $\mathrm{M}_{\mathrm{VAC}}, \mathrm{M}_{\mathrm{EMB}}, \mathrm{T}_{\mathrm{VAC}}$, and $\mathrm{T}_{\mathrm{EMB}}$; these are shown in Figure 6, along with the corresponding relative

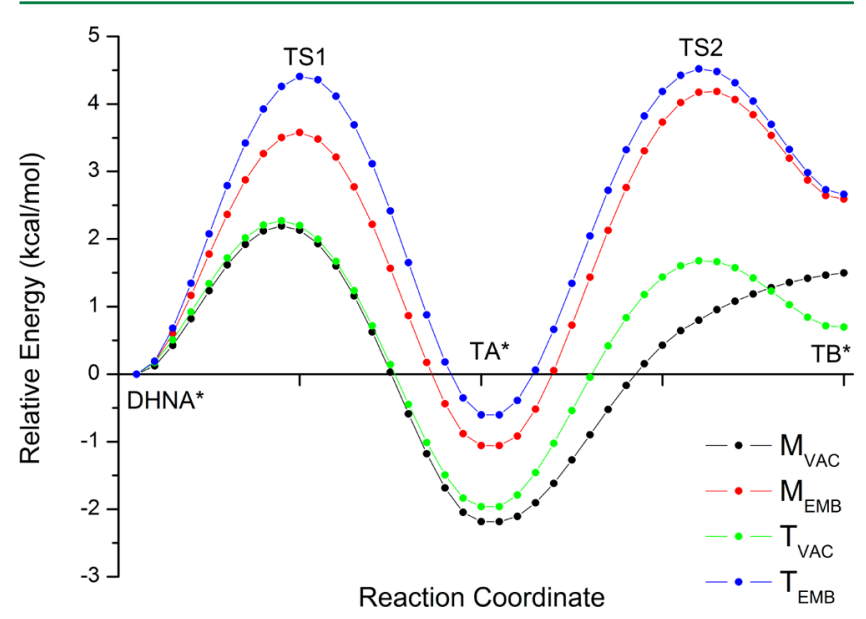

Figure 6. Excited-state potential energy surfaces mapped along a reaction coordinate interpolating between each optimized excited-state species. Each model, as outlined in Figure 5, has been considered. As $\mathrm{M}_{\mathrm{VAC}}$ did not produce an excited-state minimum for $\mathrm{TB}^{*}$, the structure for $\mathrm{M}_{\mathrm{EMB}}$ was used for interpolation.

ground-state energies at the excited-state geometry. In all cases, $\mathrm{TB}^{*}$ is higher in energy than $\mathrm{TA}^{*}$, ranging from $2.66 \mathrm{kcal} / \mathrm{mol}$ in model $\mathrm{T}_{\mathrm{VAC}}$ to $3.65 \mathrm{kcal} / \mathrm{mol}$ in model $\mathrm{T}_{\mathrm{EMB}}$. Clearly, any attempt to include the effects of the surrounding solid-state environment destabilizes the $\mathrm{TB}^{*}$ form, thereby making any emission from this species less likely. It is important to note that, as previously calculated by Peng and co-workers ${ }^{28}$ in the case of the solution, B3LYP is known to overestimate the energy of the $\mathrm{TB}^{*}$ species, giving results that are inconsistent with experimental data. In their work, this was remedied through performing single point EOM-CCSD calculations on TD-DFT optimized structures, which revealed the $\mathrm{TB}^{*}$ energy to be lower than the $\mathrm{TA}^{*}$ energy in cyclohexane solution by $1.82 \mathrm{kcal} / \mathrm{mol}$ - contrary to what was observed with B3LYP. However, given that the most reliable model $\left(\mathrm{T}_{\mathrm{EMB}}\right)$ that we have investigated so far predicts that $\mathrm{TB}^{*}$ is $3.26 \mathrm{kcal} / \mathrm{mol}$ higher in energy than $\mathrm{TA}^{*}$, it could be reasonable to conclude, despite the inherent overestimation of $\mathrm{TB}^{*}$ by B3LYP, that $\mathrm{TB}^{*}$ is indeed higher in energy than $\mathrm{TA}^{*}$ in the solid state. This is the inverse of what is observed in cyclohexane solution and so the increased emission intensity of TA*, relative to TB* in the solid state, compared with that observed in solution, is a direct result of the destabilization of the $\mathrm{TB}^{*}$ species by the crystalline environment.

Analyzing the nature of the main molecular orbitals involved in the transition (reported in Figure S2 in the Supporting Information), we can see that each step of the double proton transfer reaction results in visibly increased $\pi$-conjugation at the excited state, which was previously cited as being responsible for the reduced emission energy going from $\mathrm{TA}^{*}$ to $\mathrm{TB}^{*}$. As we have established that $\mathrm{TB}^{*}$ is likely higher in energy than $\mathrm{TA}^{*}$ in the solid state, it follows that this observation alone is not sufficient to explain the lower emission energy measured experimentally for $\mathrm{TB}^{*}$. Looking at the relative energies at the ground state for each species at the corresponding excited-state 
geometry, it can be seen that the TB ground state is significantly destabilized, relative to the TA ground state by the solid-state environment in the nonequilibrium limit (by $\sim 3.7$ and $1.8 \mathrm{kcal} / \mathrm{mol}$, comparing models $\mathrm{T}_{\mathrm{VAC}}$ and $\mathrm{T}_{\mathrm{EMB}}$ for $\mathrm{TB}$ and TA, respectively), which, in turn, reduces the emission energy. It can be seen that this effect is exaggerated upon the inclusion of the background charge distribution $\left(\mathrm{T}_{\mathrm{EMB}}\right)$ to the trimer, relative to the trimer alone $\left(\mathrm{T}_{\mathrm{VAC}}\right)$. Indeed, it is true that, in all cases where the Ewald charges are included, both $\mathrm{TA}^{*}$ and $\mathrm{TB}^{*}$ are destabilized, with $\mathrm{TB}^{*}$ being more profoundly destabilized, relative to $\mathrm{TA}^{*}$.

To further support the idea that the $\mathrm{TB}^{*}$ emission is less likely in the solid state (and therefore less intense) than the TA* emission, we will discuss the computed transition-state barrier heights at the excited state. In agreement with experimental data (and in all solid-state models tested), the TS2 barrier (corresponding to the $\mathrm{TA}^{*} \rightarrow \mathrm{TB}^{*}$ reaction) was computed to be slightly higher-ranging from $1.66 \mathrm{kcal} / \mathrm{mol}$ for model $\mathrm{M}_{\mathrm{EMB}}$ to $0.72 \mathrm{kcal} / \mathrm{mol}$ for model $\mathrm{T}_{\mathrm{EMB}}$ - than that of TS1 (corresponding to the DHNA* $\rightarrow$ TA* reaction).

Finally, in order to verify our approximation that it is sufficient to assume no cooperativity between molecules in the solid state, we reran the geometry optimization in the solid state, this time using the TA species, and re-extracted a cluster complete with the associated, recomputed Ewald charges in order to verify that there were minimal differences when compared to the initial set of calculations. The results of these calculations can be found in Table S4 in the Supporting Information, although they exhibit no significant differences (all $<0.01 \mathrm{eV}$ ), when compared to the results mentioned above.

Returning to the idea that the systematic underestimation of the $\mathrm{TA}^{*}$ and $\mathrm{TB}^{*}$ emission energies could be related to the absence of polarization of the charge embedding background, an attempt was made to alleviate this problem by, as depicted in Figure 4, using a single molecule of DHNA while calculating background charges self-consistently, with respect to the first excited states of TA and TB. This model is hereafter designated as $\mathrm{M}_{\mathrm{EMB}}^{\mathrm{SC}}$. This was done in order to mimic the mutual polarization between the central molecule and the surrounding crystalline environment after excitation of the central molecule. The results are shown in Table 1 . In this table, results with converged self-consistent charges at the previously calculated geometries for model $\mathrm{M}_{\mathrm{EMB}}$ are given. It is clear that, for both $\mathrm{TA}^{*}$ and $\mathrm{TB}^{*}$ emission, the calculation of the emission energies is significantly improved, even when compared to the best model investigated previously $\left(\mathrm{T}_{\mathrm{EMB}}\right)$. For $\mathrm{TA}^{*}$, the absolute error using model $\mathrm{T}_{\mathrm{EMB}}$ is reduced from $0.198 \mathrm{eV}(514 \mathrm{~nm})$ to $0.001 \mathrm{eV}(560 \mathrm{~nm})$, providing excellent agreement with the experimental value. In the case of $\mathrm{TB}^{*}$, where the absolute error is reduced from $0.061 \mathrm{eV}(630 \mathrm{~nm})$ to $0.047 \mathrm{eV}(634$ $\mathrm{nm}$ ), it is encouraging that the self-consistent charge method did not cause an emission energy that is already compatible with the experiment to become significantly underestimated. Furthermore, when we compare the self-consistent result with that of $\mathrm{M}_{\mathrm{VAC}}$ for $\mathrm{TB}^{*}$, we observe a significant improvement (error decreases from $0.276 \mathrm{eV}$ to $0.047 \mathrm{eV}$ ) while dealing with just a single molecule. These results show that the polarization of surrounding molecules in the molecular crystal upon the excitation of a given molecule should not be neglected when aiming to reproduce fine effects and that it could be intrinsically related to the overall photophysical properties of a molecular crystal.
From a more technical point of view, looking at both the ground-state and excited-state dipole moments for the TA and TB species allows one to rationalize the difference in red shift between $\mathrm{TA}^{*}$ and $\mathrm{TB}^{*}$ emission, with respect to solution, a phenomenon that was not recovered using the models discussed thus far. For TA, the dipole moment of the excited state $(9.03 \mathrm{D})$ was calculated to be $3.27 \mathrm{D}$ greater than that of the ground state $(5.76 \mathrm{D})$, whereas the dipole moment for TB* is only $1.08 \mathrm{D}$ greater $(7.46$ and $8.54 \mathrm{D}$ for the ground and excited states, respectively). This explains the considerably lower energy obtained with respect to the non-self-consistent charges for $\mathrm{TA}^{*}$ emission, relative to the far more modest reduction for $\mathrm{TB}^{*}$. This can be rationalized through the idea of the greater "polarizing power" of $\mathrm{TA}^{*}$, relative to $\mathrm{TB}^{*}$, on the surrounding molecules in the crystal. Indeed, the greater red shift for $\mathrm{TA}^{*}$, compared to $\mathrm{TB}^{*}$, when going from solution to the solid state is now qualitatively reproduced. It can be concluded, then, that the greater dipole moment of TA*, relative to $\mathrm{TB}^{*}$ (and, thus, the greater stabilization of $\mathrm{TA}^{*}$ in the solid state), gives rise to the apparent red shift observed experimentally for $\mathrm{TA}^{*}$ species only, going from solution in cyclohexane to the molecular crystalline environment.

Generally, it is worth noting that the charge background reaches convergence relatively quickly with a typical number of cycles ranging from 3 to 8 in this case. (For the interested reader, and to illustrate how the charges change with each step in the self-consistent process, an example is included in the Supporting Information for TA*.) Furthermore, it can be seen that, with respect to the self-consistent charges obtained for the geometry in $\mathrm{M}_{\mathrm{EMB}}$, the mutual optimization of the charges and the excited-state geometry yields no significant improvement in calculated emission energies for either TA* or TB* (see Table S3 in the Supporting Information). This suggests that, in future use of this method, one iteration-that is to say, one optimization of the charges with no subsequent geometry optimization-could be sufficient to describe the correction to the emission energies due to polarization of the surrounding crystal structure, although this requires further testing. This good agreement with experiment also lends insight into the dynamics of the excited-state process. Specifically, since all of the surrounding point charges are in the original DHNA positions and are frozen in place, this suggests that geometrical reorganization is sufficiently fast (and local) such that it can be assumed to have no effect on that of the surrounding molecules.

As a final point, it could be concluded that the stabilization of the excited states $\mathrm{TA}^{*}$ and $\mathrm{TB}^{*}$ by the polarization of the surrounding crystal lattice is, in fact, a more important factor for the proper prediction of the emission energies than the accurate descriptions of closer-range electrostatic interactions, as in the $\mathrm{QM} / \mathrm{QM}^{\prime}\left(\mathrm{T}_{\mathrm{EMB}}\right)$ approach.

\section{CONCLUSIONS}

This work has used a case study of a system (DHNA), exhibiting an excited-state intramolecular double proton transfer (ESIDPT) reaction and whose complex photophysical properties are sensitive to environmental effects, to develop and demonstrate approaches that are capable of recovering the subtle yet profound effects of a crystalline environment on photophysical properties. Initially, a background charge distribution reproducing the exact Ewald potential of the crystal was used with improved results obtained using a QM/ $\mathrm{QM}^{\prime}$ approach alongside this charge distribution. In a second 
approach, results were found to be further improved (and, indeed, in very good agreement with experiment) by using a self-consistent method to determine the values of the background point charges while considering only a single molecule explicitly at the QM level.

In this case, we demonstrate two different approaches that, while associated with very little additional computational cost, are able to retain the inherent effect of crystalline periodicity on the electrostatic potential felt by a central molecule and apply this in the context of photophysical property calculations. We have demonstrated that the inclusion of these effects can be crucial if one wishes to accurately describe and predict the result of photophysical processes at play in the solid state and, furthermore, to investigate how these may or may not differ from observed photophysical phenomena in other media, such as in solution. In the future, it could be interesting to apply this protocol to other molecular crystals with interesting photophysical properties, with particular emphasis on the use of the self-consistent charge background.

\section{ASSOCIATED CONTENT}

\section{S Supporting Information}

The Supporting Information is available free of charge on the ACS Publications website at DOI: 10.1021/acs.jctc.6b00263.

Complete Gaussian09 reference, results of periodic calculations, computed Mulliken charges of atoms in the unit cell, results from using surrounding atoms optimized at TA geometry, example of self-consistent charges for $\mathrm{TA}^{*}$ state (PDF)

\section{AUTHOR INFORMATION}

\section{Corresponding Authors}

*E-mail: frederic.labat@chimie-paristech.fr (F. Labat). *E-mail: ilaria.ciofini@chimie-paristech.fr (I. Ciofini).

\section{Notes}

The authors declare no competing financial interest.

\section{ACKNOWLEDGMENTS}

This project has received funding from the European Research Council (ERC) under the European Union's Horizon 2020 Research and Innovation Programme (Grant Agreement No. 648558).

\section{REFERENCES}

(1) Sagara, Y.; Kato, T. Mechanically Induced Luminescence Changes in Molecular Assemblies. Nat. Chem. 2009, 1, 605-610.

(2) Padalkar, V. S.; Seki, S. Excited-State Intramolecular ProtonTransfer (ESIPT)-Inspired Solid State Emitters. Chem. Soc. Rev. 2016, 45, 169-202.

(3) Gong, Y.; Zhao, L.; Peng, Q.; Fan, D.; Yuan, W. Z.; Zhang, Y.; Tang, B. Z. Crystallization-Induced Dual Emission from Metal- and Heavy Atom-Free Aromatic Acids and Esters. Chem. Sci. 2015, 6, $4438-4444$

(4) Hong, Y.; Lam, J. W. Y.; Tang, B. Z. Aggregation-Induced Emission: Phenomenon, Mechanism and Applications. Chem. Commun. 2009, 29, 4332-4353.

(5) Bertoni, R.; Cammarata, M.; Lorenc, M.; Matar, S. F.; Létard, J.F.; Lemke, H. T.; Collet, E. Ultrafast Light-Induced Spin-State Trapping Photophysics Investigated in $\mathrm{Fe}(\mathrm{phen})_{2}(\mathrm{NCS})_{2}$ SpinCrossover Crystal. Acc. Chem. Res. 2015, 48, 774-781.

(6) Presti, D.; Labat, F.; Pedone, A.; Frisch, M. J.; Hratchian, H. P.; Ciofini, I.; Menziani, M. C.; Adamo, C. Computational Protocol for
Modeling Thermochromic Molecular Crystals: Salicylidene Aniline as a Case Study. J. Chem. Theory Comput. 2014, 10, 5577-5585.

(7) Irie, M. Photochromism of Diarylethene Molecules and Crystals. Proc. Jpn. Acad., Ser. B 2010, 86, 472-483.

(8) Chevreux, S.; Paulino Neto, R.; Allain, C.; Nakatani, K.; Jacques, P.; Ciofini, I.; Lemercier, G. Solvent-Tuned Dual Emission: A Structural and Electronic Interplay Highlighting a Novel Planar. Phys. Chem. Chem. Phys. 2015, 17, 7639-7642.

(9) Allendorf, M. D.; Bauer, C. a.; Bhakta, R. K.; Houk, R. J. T. Luminescent Metal-organic Frameworks. Chem. Soc. Rev. 2009, 38, $1330-1352$.

(10) Guido, C. A.; Jacquemin, D.; Adamo, C.; Mennucci, B. Electronic Excitations in Solution: The Interplay between State Specific Approaches and a TD-DFT Description. J. Chem. Theory Comput. 2015, 11, 5782-5790.

(11) Scalmani, G.; Frisch, M. J.; Mennucci, B.; Tomasi, J.; Cammi, R.; Barone, V. Geometries and Properties of Excited States in the Gas Phase and in Solution: Theory and Application of a Time-Dependent Density Functional Theory Polarizable Continuum Model. J. Chem. Phys. 2006, 124, 094107.

(12) Miertuš, S.; Scrocco, E.; Tomasi, J. Electrostatic Interaction of a Solute with a Continuum. A Direct Utilizaion of $A b$ Initio Molecular Potentials for the Prevision of Solvent Effects. Chem. Phys. 1981, 55, $117-129$.

(13) Bernasconi, L.; Tomić, S.; Ferrero, M.; Rérat, M.; Orlando, R.; Dovesi, R.; Harrison, N. M. First-Principles Optical Response of Semiconductors and Oxide Materials. Phys. Rev. B: Condens. Matter Mater. Phys. 2011, 83, 1-7.

(14) Ferrero, M.; Rérat, M.; Orlando, R.; Dovesi, R. The Calculation of Static Polarizabilities of 1-3D Periodic Compounds. The Implementation in the CRYSTAL Code. J. Comput. Chem. 2008, 29, $1450-1459$

(15) Ferrari, A. M.; Orlando, R.; Rérat, M. Ab Initio Calculation of the Ultraviolet-Visible (UV-Vis) Absorption Spectrum, Electron-Loss Function, and Reflectivity of Solids. J. Chem. Theory Comput. 2015, 11, $3245-3258$.

(16) Walter, M.; Häkkinen, H.; Lehtovaara, L.; Puska, M.; Enkovaara, J.; Rostgaard, C.; Mortensen, J. J. Time-Dependent Density-Functional Theory in the Projector Augmented-Wave Method. J. Chem. Phys. 2008, 128, 244101.

(17) Malcıŏlu, O. B.; Gebauer, R.; Rocca, D.; Baroni, S. turboTDDFT-A Code for the Simulation of Molecular Spectra Using the Liouville-Lanczos Approach to Time-Dependent DensityFunctional Perturbation Theory. Comput. Phys. Commun. 2011, 182, $1744-1754$

(18) Presti, D.; Labat, F.; Pedone, A.; Frisch, M. J.; Hratchian, H. P.; Ciofini, I.; Cristina Menziani, M.; Adamo, C. Modeling Emission Features of Salicylidene Aniline Molecular Crystals: A QM/QM' Approach. J. Comput. Chem. 2016, 37, 861-870.

(19) Jacquemin, D.; Mennucci, B.; Adamo, C. Excited-State Calculations with TD-DFT: From Benchmarks to Simulations in Complex Environments. Phys. Chem. Chem. Phys. 2011, 13, 16987.

(20) Le Guennic, B.; Borshch, S.; Robert, V. Prussian Blue Analogue $\mathrm{CsFe}\left[\mathrm{Cr}(\mathrm{CN})_{6}\right]$ as a Matrix for the $\mathrm{Fe}(\mathrm{II})$ Spin-Crossover. Inorg. Chem. 2007, 46, 11106-11111.

(21) Kepenekian, M.; Robert, V.; Le Guennic, B.; De Graaf, C. Energetics of $\left[\mathrm{Fe}(\mathrm{NCH})_{6}\right]^{2+}$ Via CASPT2 Calculations: A SpinCrossover Perspective. J. Comput. Chem. 2009, 30, 2327-2333.

(22) Weber, J.; Schmedt Auf der Günne, J. Calculation of NMR Parameters in Ionic Solids by an Improved Self-Consistent Embedded Cluster Method. Phys. Chem. Chem. Phys. 2010, 12, 583-603.

(23) Stueber, D.; Guenneau, F. N.; Grant, D. M. The Calculation of ${ }^{13} \mathrm{C}$ Chemical Shielding Tensors in Ionic Compounds Utilizing Point Charge Arrays Obtained from Ewald Lattice Sums. J. Chem. Phys. 2001, 114, 9236-9243.

(24) Ciofini, I.; Illas, F.; Adamo, C. Performance of the $\tau$-Dependent Functionals in Predicting the Magnetic Coupling of Ionic Antiferromagnetic Insulators. J. Chem. Phys. 2004, 120, 3811-3816. 
(25) Derenzo, S. E.; Klintenberg, M. K.; Weber, M. J. Determining Point Charge Arrays That Produce Accurate Ionic Crystal Fields for Atomic Cluster Calculations. J. Chem. Phys. 2000, 112, 2074.

(26) Savarese, M.; Netti, P. A.; Adamo, C.; Rega, N.; Ciofini, I. Exploring the Metric of Excited State Proton Transfer Reactions. J. Phys. Chem. B 2013, 117, 16165-16173.

(27) Wilbraham, L.; Savarese, M.; Rega, N.; Adamo, C.; Ciofini, I. Describing Excited State Intramolecular Proton Transfer in Dual Emissive Systems: A Density Functional Theory Based Analysis. J. Phys. Chem. B 2015, 119, 2459-2466.

(28) Peng, C.-Y.; Shen, J.-Y.; Chen, Y.-T.; Wu, P.-J.; Hung, W.-Y.; Hu, W.-P.; Chou, P.-T. Optically Triggered Stepwise Double-Proton Transfer in an Intramolecular Proton Relay: A Case Study of 1,8Dihydroxy-2-Naphthaldehyde. J. Am. Chem. Soc. 2015, 137, 1434914357.

(29) Dovesi, R.; Orlando, R; Erba, A.; Zicovich-Wilson, C. M.; Civalleri, B.; Casassa, S.; Maschio, L.; Ferrabone, M.; De La Pierre, M.; D’Arco, P.; Noel, Y.; Causà, M.; Rérat, M.; Kirtman, B. CRYSTAL14: A Program for the $A b$ Initio Investigation of Crystalline Solids. Int. J. Quantum Chem. 2014, 114, 1287-1317.

(30) Vosko, S. H.; Wilk, L.; Nusair, M. Accurate Spin-Dependent Electron Liquid Correlation Energies for Local Spin Density Calculations: A Critical Analysis. Can. J. Phys. 1980, 58, 1200-1211.

(31) Lee, C.; Yang, W.; Parr, R. G. Development of the ColleSalvetti Correlation-Energy Formula into a Functional of the Electron Density. Phys. Rev. B: Condens. Matter Mater. Phys. 1988, 37, 785-789.

(32) Becke, A. D. Density-Functional thermochemistry. III. The Role of Exact Exchange. J. Chem. Phys. 1993, 98, 5648.

(33) Grimme, S. Semiempirical GGA-Type Density Functional Constructed with a Long-Range Dispersion Correction. J. Comput. Chem. 2006, 27, 1787-1799.

(34) Klintenberg, M.; Derenzo, S. E.; Weber, M. J. Accurate Crystal Fields for Embedded Cluster Calculations. Comput. Phys. Commun. 2000, 131, 120-128.

(35) Frisch, M. J.; Trucks, G. W.; Schlegel, H. B.; Scuseria, G. E.; Robb, M. A.; Cheeseman, J. R.; Scalmani, G.; Barone, V.; Mennucci, B.; Petersson, G. A.; Nakatsuji, H.; Caricato, M.; Li, X.; Hratchian, H. P.; Izmaylov, A. F.; Bloino, J.; Zheng, G.; Sonnenberg, J. L.; Hada, M.; Ehara, M.; Toyota, K.; Fukuda, R.; Hasegawa, J.; Ishida, M.; Nakajima, T.; Honda, Y.; Kitao, O.; Nakai, H.; Vreven, T.; Montgomery, J. A., Jr.; Peralta, J. E.; Ogliaro, F.; Bearpark, M.; Heyd, J. J.; Brothers, E.; Kudin, K. N.; Staroverov, V. N.; Kobayashi, R.; Normand, J.; Raghavachari, K.; Rendell, A.; Burant, J. C.; Iyengar, S. S.; Tomasi, J.; Cossi, M.; Rega, N.; Millam, J. M.; Klene, M.; Knox, J. E.; Cross, J. B.; Bakken, V.; Adamo, C.; Jaramillo, J.; Gomperts, R.; Stratmann, R. E.; Yazyev, O.; Austin, A. J.; Cammi, R.; Pomelli, C.; Ochterski, J. W.; Martin, R. L.; Morokuma, K.; Zakrzewski, V. G.; Voth, G. A.; Salvador, P.; Dannenberg, J. J.; Dapprich, S.; Daniels, A. D.; Farkas, Ö.; Foresman, J. B.; Ortiz, J. V.; Cioslowski, J.; Fox, D. J. Gaussian 09; Gaussian, Inc.: Wallingford, CT, 2009.

(36) Dapprich, S.; Komáromi, I.; Byun, K. S.; Morokuma, K.; Frisch, M. J. A New ONIOM Implementation in Gaussian98. Part I. The Calculation of Energies, Gradients, Vibrational Frequencies and Electric Field Derivatives. J. Mol. Struct.: THEOCHEM 1999, 461462, 1-21.

(37) Pisani, C.; Corà, F.; Nada, R.; Orlando, R. Hartree-Fock Perturbed-Cluster Treatment of Local Defects in Crystals. I. The EMBED Program: General Features. Comput. Phys. Commun. 1994, 82, 139-156. 